Research Paper

\title{
PARPI gene polymorphisms and neuroblastoma susceptibility in Chinese children
}

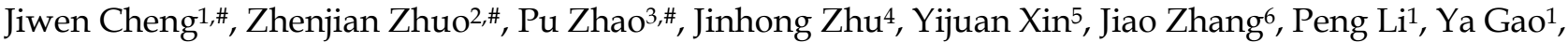 \\ Jing $\mathrm{He}^{2,}$, , Baijun Zheng1, ${ }^{1,}$ \\ 1. Department of Pediatric Surgery, the Second Affiliated Hospital of Xi' an Jiaotong University, Xi' an 710004, Shaanxi, China \\ 2. Department of Pediatric Surgery, Guangzhou Institute of Pediatrics, Guangdong Provincial Key Laboratory of Research in Structural Birth Defect Disease, \\ Guangzhou Women and Children's Medical Center, Guangzhou Medical University, Guangzhou 510623, Guangdong, China \\ 3. Department of Neonatology, Shaanxi Provincial People's Hospital, Xi'an 710068, Shaanxi, China \\ 4. Department of Clinical Laboratory, Molecular Epidemiology Laboratory, Harbin Medical University Cancer Hospital, Harbin 150040, Heilongiiang, China \\ 5. Clinical Laboratory Medicine Center of PLA, Xijing Hospital, Air Force Medical University, Xi'an 710032, Shaanxi, China \\ 6. Department of Pediatric Surgery, the First Affiliated Hospital of Zhengzhou University, Zhengzhou 450052, Henan, China
}

\#These authors contribute equally to this work.

$\square$ Corresponding authors: Baijun Zheng, Department of Pediatric Surgery, the Second Affiliated Hospital of Xi'an Jiaotong University, No. 157 West 5 Road, Xi'an 710004, Shaanxi, China, E-mail: xazbj@163.com; or Jing He, Department of Pediatric Surgery, Guangzhou Institute of Pediatrics, Guangdong Provincial Key Laboratory of Research in Structural Birth Defect Disease, Guangzhou Women and Children's Medical Center, Guangzhou Medical University, 9 Jinsui Road, Guangzhou 510623, Guangdong, China, Email: hejing198374@gmail.com.

() The author(s). This is an open access article distributed under the terms of the Creative Commons Attribution License (https://creativecommons.org/licenses/by/4.0/). See http://ivyspring.com/terms for full terms and conditions.

Received: 2019.02.18; Accepted: 2019.06.02; Published: 2019.07.10

\begin{abstract}
Neuroblastoma is a heterogeneous cancer frequently occurring in childhood. Germline mutations of PARPI oncogene are implicated in several types of cancer. However, whether common single nucleotide polymorphisms (SNPs) in PARPI gene are associated with neuroblastoma risk has received relatively few attentions. In this multi-center study, we aimed to elucidate the contributing role of PARPI SNPs in neuroblastoma risk. We successfully genotyped three potentially functional PARPI SNPs (rs1 136410 A>G, rs2666428 T>C, rs8679 A>G) in 469 neuroblastoma cases and 998 controls. We did not detect any significant association between the analyzed SNPs and neuroblastoma risk in single SNP analysis. However, stratified analysis revealed that rs 1136410 AG/GG carriers were more likely to develop tumors arising from mediastinum (AG/GG vs. AA: adjusted $\mathrm{OR}=1.65,95 \% \mathrm{Cl}=1.06-2.56, P=0.028)$. Moreover, $r \mathrm{~s} 2666428 \mathrm{TC} / \mathrm{CC}$ carriers were at significantly lower risk to develop tumors from "other sites" (TC/CC vs. TT: adjusted OR=0.44, $95 \% \mathrm{Cl}=0.20-0.96, P=0.040$ ). Our findings failed to provide evidence of the conferring role of the PARPI gene polymorphisms in the risk of neuroblastoma. Further investigations of the association between PARPI gene SNPs and neuroblastoma risk are warranted.
\end{abstract}

Key words: PARP1; polymorphism; neuroblastoma; susceptibility; DNA repair

\section{Introduction}

Neuroblastoma, arising from neural crest progenitor cells, is one of the most common extracranial solid tumors [1, 2]. Neuroblastoma mainly affects children under age of five years. Neuroblastoma accounts for about $8-10 \%$ of childhood malignancies and is related to $12-15 \%$ of cancer related childhood mortality [3-5]. The clinical patterns of neuroblastoma vary widely, ranging from spontaneous regression to relapse and therapy-resistant disease [6]. Neuroblastoma is classified into low-, intermediate-, and high-risk group [7], and high-risk neuroblastoma constitutes approximately $50 \%$ of cases [8]. Despite intensive and multi-modal therapy, 5-year survival rates of high-risk neuroblastoma patients were less than $40 \%$ [9]. One major characteristic of these high-risk neuroblastomas are their metastases to bone, noncontiguous lymph nodes, and bone marrow [2]. 
Neuroblastoma is a complex disease resulting from the interactions of environmental and genetic risk factors. Environmental factors such as maternal medication, childhood infections, and poor living habitat might increase the risk of developing neuroblastoma $[10,11]$. However, only a small portion of the exposed children finally develop neuroblastoma, suggesting the involvement of other risk factors. Growing evidence indicates that genetic factors also play an important role in the initiation and development of neuroblastoma [12, 13]. For instance, $P H O X 2 B$ and $A L K$ gene mutations contributed to tumorigenesis of neuroblastoma in a fraction of patients [14-16]. Moreover, candidate gene approaches also identified polymorphisms in NEFL [17] and CDKN1B [18] genes significantly associated with neuroblastoma susceptibility. More analysis methods, such as fine mapping, also identified neuroblastoma-associated functional risk SNPs in BARD1 [19].

DNA repair pathways are critical mechanisms for maintaining genome integrity [20]. Of DNA repair pathways, base excision repair (BER) is the major repair mechanism to repair DNA damage caused by ionizing radiation or chemical alterations of a single base $[21,22]$. The human poly(ADP-ribose) polymerase-1 (PARP1) is a key protein in BER system, recognizing DNA double strand break [23]. The activation of PARP1 is one of the early responses to DNA damage [24]. PARP1 catalyzes poly(ADPribosyl)ation, a quick DNA-damage dependent posttranslational self modification, as well as modification of histones and other nuclear proteins. PARP1 protein is essential in mediating several critical cellular mechanisms, including DNA damage recognition, DNA damage repair, mitotic apparatus function and cell death pathways [25, 26]. A number of single nucleotide polymorphisms (SNPs) in the PARP1 gene are reported to be associated with the risk of several types of cancer [27]. However, till now, no reports have evaluated the association of PARP1 gene polymorphisms with neuroblastoma risk. Thus, we performed a multi-center case-control study to explore whether PARP1 gene polymorphisms could predispose to neuroblastoma risk.

\section{Materials and Methods}

\section{Study populations}

In total, 469 patients with neuroblastoma were enrolled in three independent medical centers in China from September 2009 to March 2018, without receiving prior therapy. The three participating centers are as follows: Guangzhou Women and Children's Medical Center, Guangdong province (275 cases and 531 controls) [28-30]; The First Affiliated Hospital of Zhengzhou University, Henan province (118 cases and 281 controls) [31]; Second Affiliated Hospital of Xi'an Jiao Tong University, Shaanxi province (76 cases and 186 controls) [32]. During the same period, 998 controls were recruited from the three same geographical regions, respectively, and frequency-matched to the cases according to age $( \pm 5$ years) and gender. After providing signed informed consent, all the participants' parents were personally interviewed to collect clinical information. Blood was drawn from each participant for DNA extraction and genotyping. Details of the selection criteria have been described previously [33-35]. This study was approved by the Institutional Review Board of the three participating hospitals.

\section{SNP selection}

SNPs with a minor allele frequency (MAF) $>5 \%$ of Chinese Han in the PARP1 gene were selected from NCBI dbSNP database. An online software SNPinfo (https://snpinfo.niehs.nih.gov/) was adopted to predict putative functional potential of SNPs [36]. In final, three potentially functional SNPs (rs1136410 $A>G, r s 2666428 \mathrm{~T}>\mathrm{C}, \mathrm{rs} 8679 \mathrm{~A}>\mathrm{G}$ ) in the PARP1 gene were chosen for analysis. The rs1136410 A>G might affect splicing activity, rs2666428 $\mathrm{T}>\mathrm{C}$ and rs8679 A $>G$ might affect the microRNA binding site activity. There is no significant linkage disequilibrium $\left(R^{2}<0.8\right)$ among these three selected polymorphisms in the PARP1 gene $\left(\mathrm{R}^{2}=0.051\right.$ between $\mathrm{rs} 8679$ and $\mathrm{rs} 1136410$; $\mathrm{R}^{2}=0.26$ between $\mathrm{rs} 8679$ and $\mathrm{rs} 2666428 ; \mathrm{R}^{2}=0.196$ between rs1136410 and rs2666428) (Supplemental Figure 1).

\section{Genotyping}

Genomic DNA derivation from EDTAperipheral blood was conducted using TIANamp Blood DNA Kit (TianGen Biotech Co. Ltd., Beijing, China). The SNPs were genotyped using TaqMan platform (Applied Biosystems) with a 384-well plate [37-40]. Investigators were blind to case-control status of the study samples. For the purpose of quality control, eight water-only negative controls were used in each plate. About $10 \%$ of samples were selected randomly to be genotyped a second time, and the concordance rate was $100 \%$.

\section{Statistical analysis}

The deviation of the SNP genotypes from the Hardy-Weinberg equilibrium was assessed using the goodness-of-fit $X^{2}$ test in controls. Two-sided chi-square test was conducted to compare the distribution of demographic variables and allele frequencies between the two groups. Odds ratios (ORs), 95\% confidence intervals (CIs) and $P$ values 
were calculated. Testing for association between SNPs and neuroblastoma risk was performed using unconditional logistic regression analysis with adjustment for age and gender. Stratified analyses were also conducted by age, gender, tumor sites, and clinical stages. Analyses were carried out using the version 9.4 SAS software (SAS Institute, Cary, NC). The significant threshold was $P<0.05$ (two-sided).

\section{Results}

\section{Correlation of PARPI gene polymorphisms with neuroblastoma susceptibility}

The details of the demographic characteristics for combined subjects and Shaanxi subjects are summarized in Supplemental Table 1, while information for Guangdong and Henan subjects were described in our former publication [29-31]. Table 1 presents the genotype frequencies of PARP1 gene polymorphisms in cases and controls and their association with neuroblastoma susceptibility. The genotype frequency distribution for all SNPs were conformed to Hardy-Weinberg equilibrium (rs1136410, $P=0.669 ;$ rs2666428, $P=0.569 ; \quad$ rs8679, $P=0.256$ ). Our results indicated that participants harboring the three PARP1 SNPs were not associated with risk of neuroblastoma in Chinese population, either in single or combined genotype analysis.

\section{Stratification analysis}

We further investigated the effects of three PARP1 SNPs on the neuroblastoma risk after stratified by age, gender, tumor sites, and INSS stages (Table 2). We detected that the rs1136410 AG/GG genotypes were significantly associated with an increased risk of a tumor originating in the mediastinum (AG/GG vs. AA: adjusted $\mathrm{OR}=1.65$, 95\% $\mathrm{CI}=1.06-2.56, P=0.028)$. As to the rs2666428, negative association was detected in tumor that develops from others (TC/CC vs. TT: adjusted $\mathrm{OR}=0.44,95 \% \mathrm{CI}=0.20-0.96, P=0.040$ ). No significant association was observed in the stratified analysis of rs8679 and combined risk genotypes.

\section{Discussion}

In the current study, we comprehensively evaluated the association between the genetic variants in PARP1 gene and neuroblastoma risk. Our data reveal no significant association between the SNPs in PARP1 gene and the risk of neuroblastoma. To our knowledge this is the first evaluation of PARP1 SNPs in relation to neuroblastoma risk.

The human PARP1 gene, also known as ADPRT, is located at chromosome 1q41-42 [41]. It spans $47.3 \mathrm{~kb}$ and consists of 23 exons. Its encoding protein, PARP1, consists of three domains: an N-terminal DNA binding domain, a central auto-modification domain and a C-terminal catalytic domain $[42,43]$.

Table 1. Association between PARPI gene polymorphisms and neuroblastoma risk

\begin{tabular}{|c|c|c|c|c|c|c|c|}
\hline Genotype & Cases $(\mathrm{N}=469)$ & Controls $(\mathrm{N}=998)$ & $P$ a & Crude OR $(95 \% \mathrm{CI})$ & $P$ & Adjusted OR $(95 \% \mathrm{CI})^{\mathrm{b}}$ & $P^{b}$ \\
\hline \multicolumn{8}{|c|}{ rs1136410 (HWE=0.669) } \\
\hline AA & $136(29.00)$ & $330(33.07)$ & & 1.00 & & 1.00 & \\
\hline AG & $244(52.03)$ & $482(48.30)$ & & $1.23(0.96-1.58)$ & 0.110 & $1.23(0.95-1.58)$ & 0.116 \\
\hline GG & $89(18.98)$ & $186(18.64)$ & & $1.16(0.84-1.60)$ & 0.363 & $1.17(0.84-1.61)$ & 0.353 \\
\hline Additive & & & 0.276 & $1.09(0.94-1.28)$ & 0.260 & $1.10(0.94-1.28)$ & 0.255 \\
\hline Dominant & $333(71.00)$ & $668(66.93)$ & 0.119 & $1.21(0.95-1.54)$ & 0.119 & $1.21(0.95-1.53)$ & 0.122 \\
\hline Recessive & $380(81.02)$ & $812(81.36)$ & 0.877 & $1.02(0.77-1.35)$ & 0.876 & $1.03(0.78-1.36)$ & 0.846 \\
\hline \multicolumn{8}{|c|}{ rs2666428 (HWE=0.569) } \\
\hline TT & $305(65.03)$ & $636(63.73)$ & & 1.00 & & 1.00 & \\
\hline TC & 149 (31.77) & $325(32.57)$ & & $0.96(0.75-1.21)$ & 0.710 & $0.96(0.75-1.21)$ & 0.712 \\
\hline $\mathrm{CC}$ & $15(3.20)$ & $37(3.71)$ & & $0.85(0.46-1.56)$ & 0.593 & $0.85(0.46-1.57)$ & 0.600 \\
\hline Additive & & & 0.827 & $0.94(0.77-1.15)$ & 0.561 & $0.94(0.77-1.15)$ & 0.566 \\
\hline Dominant & $164(34.97)$ & $362(36.27)$ & 0.627 & $0.95(0.75-1.19)$ & 0.628 & $0.95(0.75-1.19)$ & 0.631 \\
\hline Recessive & $454(96.80)$ & $961(96.29)$ & 0.623 & $0.86(0.47-1.58)$ & 0.623 & $0.86(0.47-1.59)$ & 0.630 \\
\hline \multicolumn{8}{|c|}{ rs8679 $(\mathrm{HWE}=0.256)$} \\
\hline AA & $407(86.78)$ & $871(87.27)$ & & 1.00 & & 1.00 & \\
\hline AG & $60(12.79)$ & $125(12.53)$ & & $1.03(0.74-1.43)$ & 0.873 & $1.03(0.74-1.43)$ & 0.872 \\
\hline GG & $2(0.43)$ & $2(0.20)$ & & $2.14(0.30-15.25)$ & 0.448 & $2.18(0.31-15.55)$ & 0.437 \\
\hline Additive & & & 0.732 & $1.06(0.78-1.45)$ & 0.710 & $1.06(0.78-1.46)$ & 0.705 \\
\hline Dominant & $62(13.22)$ & $127(12.73)$ & 0.792 & $1.05(0.76-1.45)$ & 0.791 & $1.05(0.75-1.45)$ & 0.789 \\
\hline Recessive & $467(99.57)$ & $996(99.80)$ & 0.439 & $2.13(0.30-15.19)$ & 0.450 & $2.17(0.31-15.49)$ & 0.438 \\
\hline \multicolumn{8}{|c|}{ Combined effect of risk genotypes ${ }^{c}$} \\
\hline $0-1$ & $115(24.52)$ & $281(28.16)$ & & 1.00 & & 1.00 & \\
\hline $2-3$ & $354(75.48)$ & $717(71.84)$ & 0.144 & $1.21(0.94-1.55)$ & 0.144 & $1.21(0.94-1.55)$ & 0.148 \\
\hline
\end{tabular}

OR, odds ratio; CI, confidence interval; HWE, Hardy-Weinberg equilibrium.

a $\chi^{2}$ test for genotype distributions between neuroblastoma patients and cancer-free controls.

$\mathrm{b}$ Adjusted for age and gender.

c Risk genotypes were rs1136410 AG/GG, rs2666428 TC/TT and rs8679 AG/GG. 
Table 2. Stratification analysis for association between PARPI gene genotypes and neuroblastoma susceptibility

\begin{tabular}{|c|c|c|c|c|c|c|c|c|c|c|c|c|c|c|c|c|}
\hline \multirow[t]{2}{*}{ Variables } & \multicolumn{2}{|c|}{$\begin{array}{l}\text { rs1136410 } \\
\text { (case/control) }\end{array}$} & \multirow{2}{*}{$\begin{array}{l}\text { AOR (95\% } \\
\text { CI) }\end{array}$} & \multirow[t]{2}{*}{$P a$} & \multicolumn{2}{|c|}{$\begin{array}{l}\text { rs2666428 } \\
\text { (case/control) }\end{array}$} & \multirow[t]{2}{*}{$\begin{array}{l}\text { AOR }(95 \% \\
\text { CI })^{\mathrm{a}}\end{array}$} & \multirow[t]{2}{*}{$P a$} & \multicolumn{2}{|c|}{$\begin{array}{l}\text { rs8679 } \\
\text { (case/control) }\end{array}$} & \multirow{2}{*}{$\begin{array}{l}\text { AOR (95\% } \\
\text { CI) }\end{array}$} & \multirow[t]{2}{*}{$P a$} & \multicolumn{2}{|c|}{$\begin{array}{l}\text { Risk genotypes } \\
\text { (case/control) }\end{array}$} & \multirow[t]{2}{*}{$\begin{array}{l}\text { AOR }(95 \% \\
\text { CI })^{\mathrm{a}}\end{array}$} & \multirow[t]{2}{*}{$P a$} \\
\hline & AA & AG/GG & & & $\mathrm{TT}$ & $\mathrm{TC} / \mathrm{CC}$ & & & AA & AG/GG & & & $0-1$ & $2-3$ & & \\
\hline \multicolumn{17}{|l|}{ Age, month } \\
\hline$\leq 18$ & $53 / 128$ & $116 / 262$ & $\begin{array}{l}1.06 \\
(0.72-1.57)\end{array}$ & 0.757 & $109 / 244$ & $60 / 146$ & $\begin{array}{l}0.92 \\
(0.63-1.34)\end{array}$ & 0.650 & $141 / 348$ & $28 / 42$ & $\begin{array}{l}1.64 \\
(0.98-2.75)\end{array}$ & 0.061 & $43 / 114$ & $126 / 276$ & $\begin{array}{l}1.20 \\
(0.80-1.81)\end{array}$ & 0.381 \\
\hline$>18$ & $83 / 202$ & $217 / 406$ & $\begin{array}{l}1.30 \\
(0.96-1.77)\end{array}$ & 0.087 & $196 / 392$ & $104 / 216$ & $\begin{array}{l}0.97 \\
(0.72-1.29)\end{array}$ & 0.819 & $266 / 523$ & $34 / 85$ & $\begin{array}{l}0.80 \\
(0.52-1.23)\end{array}$ & 0.310 & $72 / 167$ & $228 / 441$ & $\begin{array}{l}1.21 \\
(0.88-1.66)\end{array}$ & 0.252 \\
\hline \multicolumn{17}{|l|}{ Gender } \\
\hline Female & $62 / 138$ & $134 / 276$ & $\begin{array}{l}1.08 \\
(0.75-1.55)\end{array}$ & 0.681 & $126 / 272$ & $70 / 142$ & $\begin{array}{l}1.07 \\
(0.75-1.52)\end{array}$ & 0.722 & $175 / 372$ & $21 / 42$ & $\begin{array}{l}1.07 \\
(0.61-1.86)\end{array}$ & 0.816 & $56 / 119$ & $140 / 295$ & $\begin{array}{l}1.01 \\
(0.69-1.47)\end{array}$ & 0.968 \\
\hline Male & $74 / 192$ & 199/392 & $\begin{array}{l}1.32 \\
(0.96-1.81)\end{array}$ & 0.091 & $179 / 364$ & $94 / 220$ & $\begin{array}{l}0.87 \\
(0.64-1.17)\end{array}$ & 0.343 & $232 / 499$ & $41 / 85$ & $\begin{array}{l}1.03 \\
(0.69-1.54)\end{array}$ & 0.896 & $59 / 162$ & $214 / 422$ & $\begin{array}{l}1.39 \\
(0.99-1.95)\end{array}$ & 0.060 \\
\hline \multicolumn{17}{|l|}{ Sites of origin } \\
\hline Adrenal gland & $52 / 330$ & $110 / 668$ & $\begin{array}{l}1.03 \\
(0.72-1.48)\end{array}$ & 0.857 & $100 / 636$ & $62 / 362$ & $\begin{array}{l}1.10 \\
(0.78-1.56)\end{array}$ & 0.571 & $144 / 871$ & $18 / 127$ & $\begin{array}{l}0.86 \\
(0.51-1.46)\end{array}$ & 0.579 & $45 / 281$ & $117 / 717$ & $\begin{array}{l}1.01 \\
(0.69-1.46)\end{array}$ & 0.972 \\
\hline Retroperitoneal & $40 / 330$ & $98 / 668$ & $\begin{array}{l}1.21 \\
(0.82-1.78)\end{array}$ & 0.351 & $89 / 636$ & $49 / 362$ & $\begin{array}{l}0.95 \\
(0.66-1.38)\end{array}$ & 0.801 & $118 / 871$ & $20 / 127$ & $\begin{array}{l}1.14 \\
(0.68-1.90)\end{array}$ & 0.614 & $32 / 281$ & $106 / 717$ & $\begin{array}{l}1.30 \\
(0.85-1.97)\end{array}$ & 0.227 \\
\hline Mediastinum & $28 / 330$ & $93 / 668$ & $\begin{array}{l}1.65 \\
(1.06-2.56)\end{array}$ & 0.028 & $78 / 636$ & $43 / 362$ & $\begin{array}{l}0.98 \\
(0.66-1.45)\end{array}$ & 0.905 & $102 / 871$ & $19 / 127$ & $\begin{array}{l}1.32 \\
(0.78-2.24)\end{array}$ & 0.296 & $24 / 281$ & $97 / 717$ & $\begin{array}{l}1.60 \\
(1.00-2.55)\end{array}$ & 0.050 \\
\hline Others & $13 / 330$ & $27 / 668$ & $\begin{array}{l}1.03 \\
(0.53-2.02)\end{array}$ & 0.931 & $32 / 636$ & $8 / 362$ & $\begin{array}{l}0.44 \\
(0.20-0.96)\end{array}$ & 0.040 & $37 / 871$ & $3 / 127$ & $\begin{array}{l}0.56 \\
(0.17-1.85)\end{array}$ & 0.343 & $12 / 281$ & $28 / 717$ & $\begin{array}{l}0.92 \\
(0.46-1.84)\end{array}$ & 0.823 \\
\hline \multicolumn{17}{|l|}{ Clinical stage } \\
\hline $\mathrm{I}+\mathrm{II}+4 \mathrm{~s}$ & $68 / 330$ & $165 / 668$ & $1.21(0.88-1.65)$ & 0.241 & $150 / 636$ & $83 / 362$ & $\begin{array}{l}0.97 \\
(0.72-1.31)\end{array}$ & 0.833 & $202 / 871$ & $31 / 127$ & $\begin{array}{l}1.07 \\
(0.70-1.63)\end{array}$ & 0.758 & $57 / 281$ & $176 / 717$ & $\begin{array}{l}1.22 \\
(0.88-1.70)\end{array}$ & 0.231 \\
\hline III+IV & $62 / 330$ & $154 / 668$ & $\begin{array}{l}1.21 \\
(0.88-1.68)\end{array}$ & 0.243 & $144 / 636$ & $72 / 362$ & $\begin{array}{l}0.88 \\
(0.64-1.20)\end{array}$ & 0.402 & $190 / 871$ & $26 / 127$ & $\begin{array}{l}0.92 \\
(0.59-1.45)\end{array}$ & 0.726 & $55 / 281$ & $161 / 717$ & $\begin{array}{l}1.13 \\
(0.81-1.58)\end{array}$ & 0.484 \\
\hline
\end{tabular}

AOR, adjusted odds ratio; $\mathrm{CI}$, confidence interval.

a Adjusted for age and gender, omitting the corresponding stratify factor.

To date, there are at least 439 SNPs found in the PARP1 gene, including 17 non-synonymous SNPs (http://www.ncbi.nlm.nih.gov/SNP). Among them, Val762Ala (rs1136410) polymorphism was one of the most investigated SNP. PARP1 Val762Ala leads to a T-to-C transition at codon 762 that causes valine to alanine amino acid substitution. Such substitution was reported to be associated with an altered activity of PARP1 protein [44, 45]. By now, several case-control studies have been performed to investigate the role of PARP1 gene Val762Ala in cancer risk [27]. Hao et al. [46] found that PARP1 Val762Ala was associated with an increased risk of esophageal squamous cell carcinoma in a case-control study of 419 patients and 480 healthy controls. In a study conducted in the USA, Lockett et al. [47] found that PARP1 Val762Ala genetic variant contributes to prostate cancer susceptibility and alters ADPRT/PARP-1 enzyme function in response to oxidative damage. In a study including 99 breast cancer cases and 96 healthy controls from Saudi population, Mohammad Alanazi et al. [48] reported for the first time that the PARP1 Ala762Ala genotype significantly contributes to breast cancer susceptibility.

Herein, we for the first time investigated whether PARP1 gene SNPs (rs1136410, rs2666428, rs8679) could affect the neuroblastoma susceptibility in Chinese children. Our results indicated no association between these three PARP1 gene SNPs and neuroblastoma risk. Several reasons might help to explain this null finding. First, the relatively small sample size might make it difficult to detect the weak association. Second, other risk factors not analyzed in the present study may be required for PARP1 gene SNPs to exert a significant effect. In a study using a bladder cancer case-control series (752 cases and 704 controls) as well as a breast cancer case-control series (257 cases and 512 controls) from UK, Mark et al. [49] found that PARP1 rs8679 was associated with increased bladder cancer and breast cancer risk. The conflicting role of rs8679 in this current study and the study conducted in UK may be ascribed to the ethnicity difference. Intriguingly, stratified analysis indicated that individuals harboring the rs1136410 AG/GG alleles were more likely to have tumor that develops from mediastinum. In the stratified analysis of rs2666428, we found a decreased association with tumor develops from others. Such conflicting role might also be the small sample size in the stratified analysis.

The study had several drawbacks. The main limitation was the relatively small sample size, which impairs our capacity to detect weak association between PARP1 gene SNPs and neuroblastoma risk. Additionally, with the available data, we could only investigate the genetic information. It is far more enough to elucidate the etiology of neuroblastoma as 
neuroblastoma is a heterogeneous disease caused by complex between genes or gene-environment interactions. Another limitation is that only three SNPs in PARP1 gene were analyzed. More potentially functional SNPs in PARP1 gene are needed to be investigated. Finally, we only included Chinese children in this study. Therefore, the conclusions obtained here should be interpreted with cautious when extrapolated to other populations.

In all, this study suggests that PARP1 polymorphisms may be weakly associated with neuroblastoma risk. Future studies are needed to validate our findings and target the functional role of PARP1 polymorphisms in neuroblastoma risk.

\section{Abbreviations}

BER: base excision repair; PARP1: poly(ADP-ribose) polymerase-1; SNP: single nucleotide polymorphism; MAF: minor allele frequency; OR: odds ratio; $\mathrm{CI}$ : confidence interval.

\section{Supplementary Material}

Supplementary figure and table. http://www.jcancer.org/v10p4159s1.pdf

\section{Acknowledgments}

This work was supported by grants from the Youth Science and Technology New Star Project of Shaanxi Province (No: 2018KJXX-050), Basic Scientific Research fee of Xi'an Jiaotong University (No: XJJ2018126 and No: YJ(ZD)201704), Pearl River S\&T Nova Program of Guangzhou (No: 201710010086) and the Guangdong Provincial Key Laboratory of Research in Structural Birth Defect Disease (No: 2019B030301004).

\section{Competing Interests}

The authors have declared that no competing interest exists.

\section{References}

1. Matthay KK, Maris JM, Schleiermacher G, Nakagawara A, Mackall CL, Diller L, et al. Neuroblastoma. Nat Rev Dis Primers. 2016; 2: 16078.

2. Capasso M, Diskin SJ. Genetics and genomics of neuroblastoma. Cancer Treat Res. 2010; 155: 65-84

3. Irwin MS, Park JR. Neuroblastoma: paradigm for precision medicine. Pediatr Clin North Am. 2015; 62: 225-56.

4. Park JR, Bagatell R, Cohn SL, Pearson AD, Villablanca JG, Berthold F, et al. Revisions to the International Neuroblastoma Response Criteria: A Consensus Statement From the National Cancer Institute Clinical Trials Planning Meeting. J Clin Oncol. 2017; 35: 2580-7.

5. Esposito MR, Aveic S, Seydel A, Tonini GP. Neuroblastoma treatment in the post-genomic era. J Biomed Sci. 2017; 24: 14.

6. Maris JM. Recent advances in neuroblastoma. N Engl J Med. 2010; 362: 2202-11.

7. Cheung NK, Dyer MA. Neuroblastoma: developmental biology, cancer genomics and immunotherapy. Nat Rev Cancer. 2013; 13: 397-411.

8. Schwab M, Westermann F, Hero B, Berthold F. Neuroblastoma: biology and molecular and chromosomal pathology. Lancet Oncol. 2003; 4: 472-80.

9. Maris JM, Hogarty MD, Bagatell R, Cohn SL. Neuroblastoma. Lancet. 2007; 369: 2106-20.
10. Cook MN, Olshan AF, Guess HA, Savitz DA, Poole C, Blatt J, et al. Maternal medication use and neuroblastoma in offspring. Am J Epidemiol. 2004; 159: 721-31.

11. Menegaux F, Olshan AF, Neglia JP, Pollock BH, Bondy ML. Day care, childhood infections, and risk of neuroblastoma. Am J Epidemiol. 2004; 159: 843-51.

12. Latorre V, Diskin SJ, Diamond MA, Zhang H, Hakonarson H, Maris JM, et al. Replication of neuroblastoma SNP association at the BARD1 locus in African-Americans. Cancer Epidemiol Biomarkers Prev. 2012; 21: 658-63.

13. Rihani A, De Wilde B, Zeka F, Laureys G, Francotte N, Tonini GP, et al. CASP8 SNP D302H (rs1045485) is associated with worse survival in MYCN-amplified neuroblastoma patients. PLoS One. 2014; 9: e114696.

14. Chen Y, Takita J, Choi YL, Kato M, Ohira M, Sanada M, et al. Oncogenic mutations of ALK kinase in neuroblastoma. Nature. 2008; 455: 971-4.

15. Mosse YP, Laudenslager M, Khazi D, Carlisle AJ, Winter CL, Rappaport E, et al. Germline PHOX2B mutation in hereditary neuroblastoma. Am J Hum Genet. 2004; 75: 727-30.

16. Mosse YP, Laudenslager M, Longo L, Cole KA, Wood A, Attiyeh EF, et al. Identification of ALK as a major familial neuroblastoma predisposition gene. Nature. 2008; 455: 930-5.

17. Capasso M, Diskin S, Cimmino F, Acierno G, Totaro F, Petrosino G, et al Common genetic variants in NEFL influence gene expression and neuroblastoma risk. Cancer Res. 2014; 74: 6913-24.

18. Capasso M, McDaniel LD, Cimmino F, Cirino A, Formicola D, Russell MR, et al. The functional variant rs34330 of CDKN1B is associated with risk of neuroblastoma. J Cell Mol Med. 2017; 21: 3224-30.

19. Cimmino F, Avitabile M, Diskin SJ, Vaksman Z, Pignataro P, Formicola D, et al. Fine mapping of $2 \mathrm{q} 35$ high-risk neuroblastoma locus reveals independent functional risk variants and suggests full-length BARD1 as tumor-suppressor. Int J Cancer. 2018; 143: 2828-37.

20. Hoeijmakers JH. Genome maintenance mechanisms for preventing cancer. Nature. 2001; 411: 366-74.

21. Krokan HE, Nilsen H, Skorpen F, Otterlei M, Slupphaug G. Base excision repair of DNA in mammalian cells. FEBS Lett. 2000; 476: 73-7.

22. Krokan HE, Bjoras M. Base excision repair. Cold Spring Harb Perspect Biol. 2013; 5: a012583.

23. Schreiber V, Dantzer F, Ame JC, de Murcia G. Poly(ADP-ribose): novel functions for an old molecule. Nat Rev Mol Cell Biol. 2006; 7: 517-28.

24. Kim MY, Zhang T, Kraus WL. Poly(ADP-ribosyl)ation by PARP-1: 'PAR-laying' NAD+ into a nuclear signal. Genes Dev. 2005; 19: 1951-67.

25. Tong WM, Yang YG, Cao WH, Galendo D, Frappart L, Shen Y, et al. Poly(ADP-ribose) polymerase-1 plays a role in suppressing mammary tumourigenesis in mice. Oncogene. 2007; 26: 3857-67.

26. Masutani M, Nakagama H, Sugimura T. Poly(ADP-ribose) and carcinogenesis. Genes Chromosomes Cancer. 2003; 38: 339-48.

27. Hua RX, Li HP, Liang YB, Zhu JH, Zhang B, Ye S, et al. Association between the PARP1 Val762Ala polymorphism and cancer risk: evidence from 43 studies. PloS One. 2014; 9: e87057.

28. He J, Zhang X, Zhang J, Zhang R, Yang T, Zhu J, et al. LMO1 super-enhancer polymorphism rs2168101 G>T correlates with decreased neuroblastoma risk in Chinese children. J Cancer. 2018; 9: 1592-7.

29. He J, Zou Y, Liu X, Zhu J, Zhang J, Zhang R, et al. Association of Common Genetic Variants in Pre-microRNAs and Neuroblastoma Susceptibility: A Two-Center Study in Chinese Children. Mol Ther Nucleic Acids. 2018; 11: 1-8.

30. Zhuo ZJ, Liu W, Zhang J, Zhu J, Zhang R, Tang J, et al. Functional Polymorphisms at ERCC1/XPF Genes Confer Neuroblastoma Risk in Chinese Children. EBioMedicine. 2018; 30: 113-9.

31. Zhang J, Zhuo Z, Li W, Zhu J, He J, Su J. XRCC1 gene polymorphisms and risk of neuroblastoma in Chinese children. Aging (Albany NY). 2018; 10: 2944-53.

32. Cheng J, Zhuo Z, Xin Y, Zhao P, Yang W, Zhou H, et al. Relevance of XPD polymorphisms to neuroblastoma risk in Chinese children: a four-center case-control study. Aging (Albany NY). 2018; 10: 1989-2000.

33. He J, Wang F, Zhu J, Zhang R, Yang T, Zou Y, et al. Association of potentially functional variants in the XPG gene with neuroblastoma risk in a Chinese population. J Cell Mol Med. 2016; 20: 1481-90.

34. He J, Yang T, Zhang R, Zhu J, Wang F, Zou Y, et al. Potentially functional polymorphisms in the LIN28B gene contribute to neuroblastoma susceptibility in Chinese children. J Cell Mol Med. 2016; 20: 1534-41.

35. He J, Zou Y, Wang T, Zhang R, Yang T, Zhu J, et al. Genetic Variations of GWAS-Identified Genes and Neuroblastoma Susceptibility: a Replication Study in Southern Chinese Children. Transl Oncol. 2017; 10: 936-41.

36. Lou J, Gong J, Ke J, Tian J, Zhang Y, Li J, et al. A functional polymorphism located at transcription factor binding sites, rs6695837 near LAMC1 gene, confers risk of colorectal cancer in Chinese populations. Carcinogenesis. 2017; 38: 177-83.

37. He J, Qiu LX, Wang MY, Hua RX, Zhang RX, Yu HP, et al. Polymorphisms in the XPG gene and risk of gastric cancer in Chinese populations. Hum Genet. 2012; 131: 1235-44.

38. Chang J, Tian J, Yang Y, Zhong R, Li J, Zhai K, et al. A Rare Missense Variant in TCF7L2 Associates with Colorectal Cancer Risk by Interacting with a GWAS-Identified Regulatory Variant in the MYC Enhancer. Cancer Res. 2018; 78: 5164-72.

39. Chang J, Zhong R, Tian J, Li J, Zhai K, Ke J, et al. Exome-wide analyses identify low-frequency variant in CYP26B1 and additional coding variants associated with esophageal squamous cell carcinoma. Nat Genet. 2018; 50: 338-43. 
40. Li J, Chang J, Tian J, Ke J, Zhu Y, Yang Y, et al. A Rare Variant P507L in TPP1 Interrupts TPP1-TIN2 Interaction, Influences Telomere Length, and Confers Colorectal Cancer Risk in Chinese Population. Cancer Epidemiol Biomarkers Prev. 2018; 27: 1029-35.

41. Rouleau M, Patel A, Hendzel MJ, Kaufmann SH, Poirier GG. PARP inhibition: PARP1 and beyond. Nat Rev Cancer. 2010; 10: 293-301.

42. Megnin-Chanet F, Bollet MA, Hall J. Targeting poly(ADP-ribose) polymerase activity for cancer therapy. Cell Mol Life Sci. 2010; 67: 3649-62.

43. Meyer-Ficca ML, Meyer RG, Jacobson EL, Jacobson MK. Poly(ADP-ribose) polymerases: managing genome stability. Int J Biochem Cell Biol. 2005; 37: 920-6.

44. Zhang X, Miao X, Liang G, Hao B, Wang Y, Tan W, et al. Polymorphisms in DNA base excision repair genes ADPRT and XRCC1 and risk of lung cancer. Cancer Res. 2005; 65: 722-6.

45. Wang XG, Wang ZQ, Tong WM, Shen Y. PARP1 Val762Ala polymorphism reduces enzymatic activity. Biochem Biophys Res Commun. 2007; 354: 122-6.

46. Hao B, Wang H, Zhou K, Li Y, Chen X, Zhou G, et al. Identification of genetic variants in base excision repair pathway and their associations with risk of esophageal squamous cell carcinoma. Cancer Res. 2004; 64: 4378-84.

47. Lockett KL, Hall MC, Xu J, Zheng SL, Berwick M, Chuang SC, et al. The ADPRT V762A genetic variant contributes to prostate cancer susceptibility and deficient enzyme function. Cancer Res. 2004; 64: 6344-8.

48. Alanazi M, Pathan AA, Abduljaleel Z, Shaik JP, Alabdulkarim HA, Semlali A, et al. Association between PARP-1 V762A polymorphism and breast cancer susceptibility in Saudi population. PLoS One. 2013; 8: e85541.

49. Teo MT, Landi D, Taylor CF, Elliott F, Vaslin L, Cox DG, et al. The role of microRNA-binding site polymorphisms in DNA repair genes as risk factors for bladder cancer and breast cancer and their impact on radiotherapy outcomes. Carcinogenesis. 2012; 33: 581-6. 